\title{
Semi-Static Hedging Based on a Generalized Reflection Principle on a Multi Dimensional Brownian Motion
}

\author{
Yuri Imamura*and Katsuya Takagi ${ }^{\dagger}$
}

\begin{abstract}
On a multi-assets Black-Scholes economy, we introduce a class of barrier options, where the knock-out boundary is a cone. In this model we apply a generalized reflection principle in a context of the finite reflection group acting on a Euclidean space to give a valuation formula and the semi-static hedge. The result is a multi-dimensional generalization of the put-call symmetry by Bowie and Carr (1994), Carr and Chou (1997), etc. The important implication of our result is that with a given volatility matrix structure of the multi-assets, one can design a multi-barrier option and a system of plain options, with the latter the former is statically hedged.
\end{abstract}

Keywords: Semi-static hedging, Barrier option, Put-call symmetry, Reflection group

\section{Introduction}

In this paper, we introduce a class of barrier options (knocked-in and knockedout options) on a multi-assets Black-Scholes economy and give a semi-static hedging technique based on a generalized reflection principle in a context of the finite reflection group acting on a Euclidean space. A semi-static hedging strategy will be obtained from an equation between the value of barrier options and that of path-independent options.

${ }^{*}$ Department of Mathematical Sciences, Ritsumeikan University. Email: yuri.imamura@gmail.com

${ }^{\dagger}$ Department of Mathematical Sciences, Ritsumeikan University. 


\subsection{The previous studies on one risky asset cases}

Before going into details, we give a short survey of the context. Merton (1973) was the first to discuss a hedging strategy of a barrier option. It was in the Black-Scholes framework. The valuation formula was in closed form but using a dynamic delta hedging strategy in the underlying asset. Bowie and Carr (1994) gave a hedging strategy of barrier options using putcall symmetry in the Black-Scholes framework. Their strategy is semi-static in that it is a portfolio of a few European options with a fixed maturity rebalanced at most one time.

The put-call symmetry is a geometric generalization of the reflection principle, which is roughly state as the following relation on the process $S$;

$$
\mathbf{E}_{0}\left[f\left(S_{T}\right) 1_{\left\{\tau_{H}>T\right\}}\right]=\mathbf{E}_{0}\left[1_{\left\{S_{T}>H\right\}} f\left(S_{T}\right)\right]-\mathbf{E}_{0}\left[1_{\left\{S_{T}<H\right\}} f\left(H^{2} / S_{T}\right)(S / H)^{p}\right]
$$

for any bounded $f$, where $\tau_{H}$ is the first hitting time of the process to the boundary $H>0$. The power $p$ is related to the drift; $p=1$ when $S$ is a martingale. One sees why it is called put-call symmetry by looking at the case $f(x)=(x-H)_{+}$. Detailed discussions, applications, and extensions are found in Bowie and Carr (1994), Carr. Ellis, and Gupta (1998), Poulsen (2006), Carr and Chou (1997) and Carr and Lee (2009).

\subsection{The multi-risky asset case}

As mentioned above, in this paper we are interested in the static hedge of knock-out options written on multi-assets. There have been several attempts to work on barrier options of multi-assets (Molchanov and Schmutz (2010), Schmutz (2010), etc) but they are basically working on one reflection; the group structure is trivial. The present paper in contrast heavily relies on the group structure.

Suppose that the prices of the assets are given by strictly positive adapted processes $S^{i}, i=1, \cdots, n$, defined on a filtered probability space $\left(\Omega, \mathcal{F}, P,\left\{\mathcal{F}_{t}\right\}\right)$. We assume that 0 -th asset is non-risky; $S^{0}$ is a strictly positive deterministic process and that $S^{i} / S^{0}, i=1,2, \cdots, n$ are strictly positive martingales. By the fundamental theorem of asset pricing, it may mean that the market is arbitrage-free and complete and $P$ is already the unique equivalent martingale measure.

The pay-off of a knock-out option in its generic form is

$$
f\left(S_{T}\right) 1_{\{\tau \geq T\}},
$$

where $f: \mathbf{R}^{n+1} \rightarrow \mathbf{R}$ is a measurable function and $\tau_{D}$ is the first exit time of the $n+1$-dimensional process $S=\left(S^{0}, S^{1}, \cdots, S^{n}\right)$ out of a region $D \subset \mathbf{R}^{n+1}$, 
which should be a stopping time with respect to the market filtration $\left\{\mathcal{F}_{t}\right\}$. Following Imamura (2011), we formulate the possibility of a static hedge as follows.

Definition 1.1. Let $g$ be a measurable function on $\mathbf{R}^{n+1}$. We say a knock-out option (1.2) is statically hedged by the path-independent option (whose pay-off is) $g$ if it holds

$$
\mathbf{E}_{0}\left[1_{\{\tau \geq T\}} f\left(S_{T}\right) \mid \mathcal{F}_{t}\right]=1_{\{\tau \geq t\}} \mathbf{E}_{0}\left[g\left(S_{T}\right) \mid \mathcal{F}_{t}\right], \quad t \in[0, T],
$$

assuming that both sides are finite.

Financially speaking, the left-hand-side (1.3) stands for the price at time $t$ of the knock-put option, while the right-hand-side is the value of the pathindependent option until the exit time $\tau$. The equality claims that the former is hedged by holding the latter until $\tau$. At the time $\tau$, both sides becomes zero, meaning in particular that the value of the path-independent option can be sold without any cost.

The main result of the present paper is Theorem 3.1, where we will establish

$$
\begin{aligned}
& \mathbf{E}_{0}\left[1_{\left\{\tau_{D_{H}}>T\right\}} f\left(S_{T}^{C}\right) \mid \mathcal{F}_{t}\right] \\
& =1_{\left\{\tau_{D_{H}}>t\right\}} \mathbf{E}_{0}\left[\sum_{w \in W(\Phi)} \varepsilon(w) f\left(H S_{T}^{C w} / H^{T_{w}}\right) S_{T}^{t} x_{w} C / H^{t} x_{w} 1_{\left\{S_{T}^{C w}>H^{T w}\right\}} \mid \mathcal{F}_{t}\right] .
\end{aligned}
$$

Here, $S$ is a given multi-dimensional geometric Brownian motion, and $H$ is a given (multi-) boundary, and $W(\Phi)$ is the reflection group; detailed descriptions to them will be given in section 2 and section 3 . The implication of (1.4) is that, given $S$, one can choose $C$ so that we can utilize the group structure of $W(\Phi)$ to construct a static hedge of the option.

In Remark 3.2, we show that Theorem 3.1 is actually a generalization of the put-call symmetry (1.1).

\subsection{The organization of the present paper}

In section 2 we first recall the Brownian motion in a fundamental domain of the action of a finite reflection group. Various descriptions of $n$-dimensional Brownian motion conditioned for its components to never collide and more generally of Brownian motion in the fundamental domain have been studied extensively in recent time, by for example Grabiner (1999), O'Connell 
(2003), Biane, Bougerol, and O'Connell (2005), Jones and O'Connell (2006) and references therein. Such a reflected Brownian motion is a generalization of the reflecting Brownian motion in $[0, \infty)$.

In Section 3, we give the main result (Theorem 3.1) of the present paper. Using the generalized reflection principle on the Brownian motion, we give semi-static hedging strategies of knock-out options in a multi-stock model where the knock-out boundary is related to a root system.

Akahori and Takagi (2010) is the earliest related paper, where only a simplest case is studied. Akahori and Imamura (2012) is the latest one, where symmetrization of diffusion processes, a technique for numerical analysis of barrier options, is introduced, where a generalization of the reflection principle, which is still based on the symmetry of reflection groups, plays a central role.

\section{Generalized reflection principle}

\subsection{The finite reflection groups}

We recall basics of the finite reflection groups from Hall (2003). Let $V$ be a real finite dimensional Euclidean space endowed with an inner product $\langle u, v\rangle$ for $u, v \in V$. A reflection is a linear operator $s_{\alpha}$ on $V$ which transforms a nonzero element $\alpha \in V$ to the one reflected with respect to the hyperplane $H_{\alpha}:=\{x \in V:\langle x, \alpha\rangle=0\}$ orthogonal to $\alpha$. The reflection is explicitly given by:

$$
s_{\alpha}(x)=x-\frac{2\langle x, \alpha\rangle}{\langle\alpha, \alpha\rangle} \alpha(x \in V) .
$$

Since we attach greater importance to an element $\alpha$ of $V$ than the hyperplane $H_{\alpha}$, we call $s_{\alpha}$ a reflection operator with respect to $\alpha$. It is easy to see that $s_{\alpha}$ is an orthogonal transformation. Since $s_{\alpha}$ move $\alpha$ to $-\alpha$, it holds that $s_{\alpha}^{2}=1$. It means $s_{\alpha}$ has order 2 in the group $O(d)$ of all orthogonal transformations of $V$. Take $\Phi$ to be a finite set such that nonzero vectors in $\Phi$ satisfying the two conditions:

(R1) $\Phi \cap \mathbf{R} \alpha=\{\alpha,-\alpha\}$ for all $\alpha \in \Phi$

(R2) $s_{\alpha} \Phi=\Phi$ for any $\alpha \in \Phi$.

Then a group $W(\Phi)$ generated by reflections $\left\{s_{\alpha}: \alpha \in \Phi\right\}$ (we call it a reflection group in short) is a finite subgroup of $O(d)$. We call $\Phi$ a root system associated with a reflection group because of the connection between Weyl groups and root systems of semi-simple Lie algebras. 
Our definition of root systems differs somewhat from that commonly used in Lie theory. A finite reflection group is characterized by our root system, and conversely, the group generated by a root system is finite.

Fix a root system $\Phi$. A fundamental system is a subset $\Sigma$ of a root system $\Phi$ that satisfies the following properties:

(F1) The elements of $\Sigma$ are linearly independent.

(F2) Every element of $\Phi$ can be written as a linear combination of the elements of $\Sigma$ with coefficients all of the same sign (all non-positive or all nonnegative).

Then the reflection group $W(\Phi)$ is a group generated by reflections $s_{\alpha}$ for $\alpha \in \Sigma$, and subject only to the relations: $\left(s_{\alpha} s_{\beta}\right)^{m(\alpha, \beta)}=1$ for $\alpha, \beta \in \Sigma$. (See, for example, Section 1.9 in Humphreys (1990).) Because of (R1), $\{w(\alpha)$ : $w \in W(\Phi), \alpha \in \Sigma\}$ is equal to the root system $\Phi$, therefore the root system is retrieved by the associated fundamental system.

For each $\alpha \in \Sigma$, we set the open half-space $A_{\alpha}:=\{x \in V:\langle x, \alpha\rangle>0\}$. As an intersection of open convex sets, $C_{\Sigma}:=\cap_{\alpha \in \Sigma} A_{\alpha}$ is an open convex set. We call $C_{\Sigma}$ a chamber corresponding to a fundamental system $\Sigma$. Let $\bar{C}_{\Sigma}$ to be the closure of $C_{\Sigma}$. Note that the hyperplane $\cup_{\alpha \in \Phi} H_{\alpha}$ is the boundary $\partial C_{\Sigma}:=\bar{C}_{\Sigma} \backslash C_{\Sigma}$. We note that a chamber $C_{\Sigma}$ is a interior set of a fundamental domain for the action of $W(\Phi)$ on $V$, that is, each $x \in V \backslash \cup_{\alpha \in \Phi} H_{\alpha}$ is conjugate under $W(\Phi)$ to one and only one element in $C_{\Sigma}$. It is clear that replacing $\Sigma$ by $\omega \Sigma$ replaces $C_{\Sigma}$ by $\omega C_{\Sigma}$ for $\omega \in W(\Phi)$. Thus the chambers are characterized topologically as the connected components of the complement in $V$ of $\cup_{\alpha \in \Phi} H_{\alpha}$. Conversely, elements of a root system can be characterized as vectors which are orthogonal to some wall of a chamber.

\subsection{Brownian motion in a chamber corresponding to a fundamental system of a root system}

Let $B=\left(B_{t}\right)_{t>0}$ be a standard Brownian motion in $\mathbf{R}^{d}$ and $\mathbf{P}_{x}$ be a Wiener measure with the initial distribution $\delta_{x}$. The transition density of Brownian motion in $\mathbf{R}^{d}$ is given by

$$
p_{t}(x, y)=(2 \pi t)^{-d / 2} \exp \left(-\frac{|y-x|^{2}}{2 t}\right), x, y \in \mathbf{R}^{d} \text { and } t \geq 0 .
$$

Note that $p_{t}$ is invariant under the action of the group $O(d)$ of all orthogonal transformations of $\mathbf{R}^{d}$ and hence a reflection group.

Fix a root system $\Phi$ and its fundamental system $\Sigma$. For a chamber $C_{\Sigma}$, let $T_{C_{\Sigma}}:=\inf \left\{t \geq 0: B_{t} \notin C_{\Sigma}\right\}$ be the first hitting time on the boundary 
$\partial C_{\Sigma}$. We denote by $\hat{p}_{t}(x, d y)$ the transition density of Brownian motion in $C_{\Sigma}$ killed at the boundary $\partial C_{\Sigma}$, that is,

$$
\hat{p}_{t}(x, A)=\mathbf{P}_{x}\left(B_{t} \in A, T_{C_{\Sigma}}>t\right)
$$

for any Borel set $A$ of $\bar{C}_{\Sigma}$. We can reconstruct $\hat{p}_{t}$ by using $p_{t}$ and a generalized reflection principle (see for details Karlin and McGregor (1959)) as:

$$
\hat{p}_{t}(x, d y)=\sum_{w \in W(\Phi)} \varepsilon(w) p_{t}(x, w(y)) d y
$$

for $x, y \in C_{\Sigma}$, where

$$
\varepsilon(w)=\operatorname{det} w= \begin{cases}1 & \text { if } w=s_{\alpha_{1}} s_{\alpha_{2}} \cdots s_{\alpha_{k}}, \text { and } k \text { is even. } \\ -1 & \text { if } w=s_{\alpha_{1}} s_{\alpha_{2}} \cdots s_{\alpha_{k}}, \text { and } k \text { is odd }\end{cases}
$$

In the following sections, we aim to apply this expression to a static hedging strategy of a knock-out option in the context of mathematical finance.

\section{The Static Hedge of Options Knocked Out at the Boundary of a Chamber Correspond- ing to a Fundamental System of a Root System}

\subsection{Knock-out Option on a multi-asset Black-Scholes type model}

We work on the following Black-Scholes economy: the price processes $S_{t}^{i}$, $i=0,1, \cdots, n$ are given by

$$
S_{t}^{i}=S_{0}^{i} \exp \left\{\left(\Lambda B_{t}\right)_{i}+\left(r-\frac{1}{2}\left(\Lambda \Lambda^{*}\right)_{i, i}\right) t\right\}, \quad S_{0}^{i}>0
$$

where $B$ is a standard $d$-dimensional $(d \leq n)$ Brownian motions starting from $0 \Lambda$ is an $(n+1) \times d$ matrix with $\operatorname{rank} \Lambda=d$, and $r$ is the risk-free interest rate. Here 0 -th row vector of $\Lambda$ is set to be zero so that we have

$$
S_{t}^{0}=S_{0}^{0} \exp (r t)
$$

Let $\Lambda_{l}$ and $\Lambda_{r}$ be a $d \times(n+1)$ matrix and a $d \times d$ matrix such that $\Lambda_{l} \Lambda=E_{d}$ and $\Lambda \Lambda_{r}=E_{r}$, respectively. Since $\operatorname{rank} \Lambda=d, \Lambda_{l}$ and $\Lambda_{r}$ are exist. 
Let $m$ be an integers smaller than $d$, and $C=\left(c_{k, i}\right)$ be an $m \times(n+1)$ matrix with $\operatorname{rank} C=m$. Define

$$
D_{H}:=\bigcap_{k=1}^{m}\left\{s=\left(s^{0}, s^{1}, \cdots, s^{n}\right) \in \mathbf{R}^{n+1}: \prod_{i=0}^{n}\left(s^{i}\right)^{c_{k, i}} \leq H_{k}\right\},
$$

where $H=\left(H_{1}, \cdots, H_{m}\right) \in \mathbf{R}_{++}^{m}$. This is not an empty set by the assumption that rank $C=m$. We study the possibility of the static hedge of the knockout options whose pay-off is $f\left(S_{T}\right) 1_{\left\{\tau_{D} \geq T\right\}}$. Recall that $\tau_{D_{H}}$ is the first exit time of $S$ out of $D_{H}$. To see if $P\left(\tau_{D_{H}}<T\right)>0$ or not, we rewrite all the settings in terms of the Brownian motion $B$. Define

$$
G:=\left\{x=\left(x^{1}, \cdots, x^{d}\right) \in \mathbf{R}^{d}: C \Lambda x \in \mathbf{R}_{+}^{m}\right\} .
$$

Note that $G$ is a convex cone $\neq \emptyset$ since $\operatorname{rank} C \Lambda=m$.

Let $\mu$ and $h$ be such that

$$
C \Lambda \mu=C\left\{r \mathbf{1}-\frac{1}{2} \operatorname{diag}\left(\Lambda \Lambda^{*}\right)\right\}
$$

and

$$
C \Lambda h=C \log S_{0}-\log H,
$$

where 1 denotes the vacuum vector $(1,1, \cdots, 1)$, and diag : $M(n+1) \rightarrow \mathbf{R}^{n+1}$ sends a matrix to the vector composed of its diagonal entries. Such $\mu$ and $h$ exist since $\operatorname{rank}(C \Lambda)=m$.

With these, we have that

$$
\tau_{D_{H}}=\inf \left\{t>0: h+B_{t}+\mu t \notin G\right\} .
$$

Now we see that $P\left(0<\tau_{D_{H}}<T\right)>0$.

\subsection{Possibility of Static Hedge}

Before stating our main result, we introduce some notations. For vectors $a$ and $b$, map $\phi: \mathbf{R} \rightarrow \mathbf{R}$ and $k \times l$ matrix $M=\left(m_{i, j}\right)$, we mean $\left(a_{1} b_{1}, \cdots, a_{k} b_{k}\right)$ by $a b,\left(a_{1} / b_{1}, \cdots, a_{k} / b_{k}\right)$ by $a / b,\left(\phi\left(a_{1}\right), \cdots, \phi\left(a_{k}\right)\right)$ by $\phi(a)$,

$$
\left(\prod_{j=1}^{k} v_{j}^{m_{1, j}}, \cdots, \prod_{j=1}^{k} v_{j}^{m_{l, j}}\right)
$$

by $\mathbf{v}^{M}$, and $a<b$ means $a_{j}<b_{j}$ for all $1 \leq j \leq k$.

Now we are in a position to state our main result. Let $\Sigma=\left\{\alpha_{1}, \alpha_{2} \cdots, \alpha_{m}\right\}$ be a fundamental system of a root system $\Phi$ on $\mathbf{R}^{d}$ with $\sharp \Sigma=m$. Let $C$ 
satisfy $C \Lambda=\Sigma^{\prime}$, where $i$-th row of the $m \times d$ matrix $\Sigma^{\prime}$ is $\nu_{i} \alpha_{i}$, for some $\left(\nu_{1}, \cdots, \nu_{m}\right) \in \mathbf{R}_{++}^{m}$. Since $\Lambda$ and $\Sigma^{\prime}$ is of full rank (the latter is implied by (F1) in section 2.1), such $C$ with $\operatorname{rank} C=m$ exists. Note that $\operatorname{span} \Sigma=C \Lambda$ is invariant under the action of $\omega \in W(\Phi)$, which is implied by (F2) in section 2.1. The representation matrix for the action by $w$ will be denoted by $T_{w} \in G L(m, \mathbf{R})$. That is,

$$
C \Lambda w=T_{w} C \Lambda \text {. }
$$

With the choice of $C$ subject to the volatility $\Lambda$ and a fundamental system $\Phi$, we have the following

Theorem 3.1. (i) The set $G$ given by (3.2) is a fundamental domain of the action of the reflection group $W(\Phi)$. (ii) In such a case, any measurable $f: \mathbf{R}_{++}^{m} \rightarrow \mathbf{R}$ with at most linear growth, $f\left(S_{T}^{C}\right) 1_{\left\{\tau_{D_{H}}>T\right\}}$ is statically hedged by

$$
\sum_{w \in W(\Phi)} \varepsilon(w) f\left(H S_{T}^{C_{w}} / H^{T_{w}}\right) S_{T}^{t} x_{w} C / H^{t} x_{w} 1_{\left\{S_{T}^{C w}>H^{\left.T_{w}\right\}}\right.}
$$

where $C_{w}=T_{w} C$ and $x_{w} \in \mathbf{R}^{m}$ is a solution to ${ }^{t} \Sigma^{\prime} x_{w}=w(\mu)-\mu$, which surely exists by the linearly independence among the roots.

Proof. By the Markov property, it suffices to show

$\mathbf{E}_{0}\left[f\left(S_{T}^{C}\right) 1_{\left\{\tau_{D_{H}}>T\right\}}\right]=\mathbf{E}_{0}\left[\sum_{w \in W(\Phi)} \varepsilon(w) f\left(H S_{T}^{C_{w}} / H^{T_{w}}\right) S_{T}^{t} x_{w} C / H^{t} x_{w} 1_{\left\{S_{T}^{C_{w}}>H^{T w}\right\}}\right]$.

Applying the Cameron-Martin-Maruyama-Girsanov theorem, we obtain that

$$
\begin{aligned}
& \mathbf{E}_{0}\left[f\left(S_{T}^{C}\right) 1_{\left\{\tau_{D_{H}}>T\right\}}\right] \\
& =\mathbf{E}_{0}\left[f\left(S_{0}^{C} e^{C\left\{\Lambda\left(B_{T}-\mu t\right)+\left(r \mathbf{1}-\frac{\operatorname{diag} \Lambda \Lambda^{*}}{2}\right) T\right\}}\right)\right. \\
& \left.\quad e^{\left\langle\mu, B_{T}\right\rangle-\frac{|\mu|^{2} T}{2}}: \inf \left\{t>0: B_{t}+h \notin G\right\}>T\right],
\end{aligned}
$$

where $\langle\cdot, \cdot\rangle$ is the Euclidean inner product and $|\cdot|$ is the Euclidean norm. By shifting the initial point of Brownian motion to $h$,

(the right hand side of (3.6))

$$
\begin{aligned}
=\mathbf{E}_{h}\left[f\left(S_{0}^{C} e^{C\left\{\Lambda\left(B_{T}-h-\mu T\right)+\left(r \mathbf{1}-\frac{\operatorname{diag} \Lambda \Lambda^{*}}{2}\right) T\right\}}\right)\right. & \\
& \left.e^{\left\langle\mu, B_{T}-h\right\rangle-\frac{|\mu|^{2} T}{2}}: \inf \left\{t>0: B_{t} \notin G\right\}>T\right] .
\end{aligned}
$$

By the expression (2.1), we have

(the right hand side of (3.7))

$$
\begin{gathered}
=\mathbf{E}_{h}\left[\sum_{\omega \in W(\Phi)} \varepsilon(w) f\left(S_{0}^{C} e^{C\left\{\Lambda\left(w\left(B_{T}\right)-h-\mu T\right)+\left(r \mathbf{1}-\frac{\operatorname{diag} \Lambda \Lambda^{*}}{2}\right) T\right\}}\right)\right. \\
\left.e^{\left\langle\mu, w\left(B_{T}\right)-h\right\rangle-\frac{|\mu|^{2} T}{2}}: w\left(B_{T}\right) \in G\right] .
\end{gathered}
$$


Since $w$ is an orthogonal transformation, $\left\langle\mu, w\left(B_{T}\right)\right\rangle$ is equal to $\left\langle w^{-1}(\mu), B_{T}\right\rangle$. We note that $w^{-1}=w$ since it is a reflection; has the order 2 . Therefore we see that

(the right hand side of (3.8))

$$
\begin{gathered}
=\mathbf{E}_{h}\left[\sum_{\omega \in W(\Phi)} \varepsilon(w) f\left(S_{0}^{C} e^{C\left\{\Lambda w B_{T}-\Lambda(h+\mu T)+\left(r \mathbf{1}-\frac{\operatorname{diag} \Lambda \Lambda^{*}}{2}\right) T\right\}}\right)\right. \\
\left.e^{\left\langle w(\mu), B_{T}\right\rangle-\langle\mu, h\rangle-\frac{|\mu|^{2} T}{2}}: w\left(B_{T}\right) \in G\right] .
\end{gathered}
$$

By shifting the initial point again, we obtain that

(the right hand side of (3.9))

$$
\begin{array}{r}
=\mathbf{E}_{0}\left[\sum_{\omega \in W(\Phi)} \varepsilon(w) f\left(S_{0}^{C} e^{C\left\{\Lambda w\left(B_{T}+h\right)-\Lambda(h+\mu T)+\left(r \mathbf{1}-\frac{\mathrm{diag} \Lambda \Lambda^{*}}{2}\right) T\right\}}\right)\right. \\
\left.e^{\left\langle w(\mu), B_{T}\right\rangle+\langle w(\mu)-\mu, h\rangle-\frac{|\mu|^{2} T}{2}}: w\left(B_{T}+h\right) \in G\right] \\
=\mathbf{E}_{0}\left[\sum_{\omega \in W(\Phi)} \varepsilon(w) f\left(S_{0}^{C} e^{C\left\{\Lambda w\left(B_{T}+h\right)-\Lambda(h+\mu T)+\left(r \mathbf{1}-\frac{\operatorname{diag} \Lambda \Lambda^{*}}{2}\right) T\right\}}\right)\right. \\
\left.e^{\left\langle\mu, B_{T}\right\rangle-\frac{|\mu|^{2} T}{2}} e^{\left\langle w(\mu)-\mu, B_{T}+h\right\rangle}: w\left(B_{T}+h\right) \in G\right] .
\end{array}
$$

Using the Cameron-Martin theorem again, we see that

(the right hand side of (3.10) )

$$
\begin{gathered}
=\mathbf{E}_{0}\left[\sum_{\omega \in W(\Phi)} \varepsilon(w) f\left(S_{0}^{C} e^{C\left\{\Lambda w\left(B_{T}+\mu T+h\right)-\Lambda(h+\mu T)+\left(r \mathbf{1}-\frac{\operatorname{diag} \Lambda \Lambda^{*}}{2}\right) T\right\}}\right)\right. \\
\left.e^{\left\langle w(\mu)-\mu, B_{T}+\mu T+h\right\rangle}: w\left(B_{T}+\mu T+h\right) \in G\right] \\
=\mathbf{E}_{0}\left[\sum_{\omega \in W(\Phi)} \varepsilon(w) f\left(H e^{C\left\{\Lambda w\left(B_{T}+\mu T+h\right)\right\}}\right)\right. \\
\left.e^{\left\langle w(\mu)-\mu, B_{T}+\mu T+h\right\rangle}: w\left(B_{T}+\mu T+h\right) \in G\right]
\end{gathered}
$$

where the last equality of (3.11) comes from (3.3) and (3.4).

Therefore, we have to show

$$
\begin{gathered}
H S_{T}^{C_{w}} / H^{T_{w}}=e^{C\left\{\Lambda w\left(B_{T}+\mu T+h\right)+\log H\right\}}, \\
S_{T}^{t} x_{w} C / H^{t} x_{w}=e^{\left\langle w(\mu)-\mu, B_{T}+\mu T+h\right\rangle},
\end{gathered}
$$

and

$$
\left\{S_{T}^{C_{w}}>H^{T_{w}}\right\}=\left\{w\left(B_{T}+\mu T+h\right) \in G\right\} .
$$


The relation (3.12) is equivalent to

$$
\begin{aligned}
C_{w} \Lambda & =C \Lambda w, \\
C_{w} \log S_{0}+\log H-\log H^{T_{w}} & =C \Lambda w h+\log H,
\end{aligned}
$$

and

$$
C_{w}\left(r \mathbf{1}-\frac{\operatorname{diag} \Lambda \Lambda^{*}}{2}\right)=C \Lambda w \mu \text {. }
$$

By the defining relation (3.5) of $C_{w},(3.15)$ is valid. Substituting (3.3), (3.4) and (3.15), we also confirm (3.16) and (3.17). Also, the relation (3.13) is equivalent to

$$
\begin{aligned}
{ }^{t} x_{w} C \Lambda & =w(\mu)-\mu \\
{ }^{t} x_{w}\left(C \log S_{0}-\log H\right) & =(w(\mu)-\mu) h
\end{aligned}
$$

and

$$
{ }^{t} x_{w} C\left(r \mathbf{1}-\frac{\operatorname{diag} \Lambda \Lambda^{*}}{2}\right)=(w(\mu)-\mu) \mu .
$$

Similarly as the case of (3.12), (3.18), (3.19) and (3.20) are valid. Finally, the right hand side of (3.14) is equal to

$$
\left\{C \Lambda w\left(B_{T}+\mu T+h\right) \in \mathbf{R}_{+}^{m}\right\}=\left\{e^{C \Lambda w\left(B_{T}+\mu T+h\right)}>\mathbf{1}\right\} .
$$

Hence (3.14) is satisfied due to (3.12).

Remark 3.2. We show that in the case of 1-dimension, Theorem 3.1 reduces to the put-call symmetry (1.1) by Bowie and Carr (1994). Suppose that in the Black-Scholes economy; there is a risky asset with volatility $\sigma$, and the interest rate is $r$. We take a fundamental system $\Sigma=\{1\}$ on $\mathbf{R}$. Then the root system generated by $\Sigma$ is $\Phi=\{1,-1\}$, and the reflection group is $W(\Phi)=\left\{1, s_{1}\right\}$, where we recall that $s_{1}(x)=-x$ for $x \in \mathbf{R}$. Then we obtain that $T_{1} x=x$ and $T_{s_{1}} x=-x$ for $x \in \mathbf{R}$. We take $\nu=1$. Then $C=1, \mu=r-\frac{1}{2} \sigma^{2}, x_{1}=0$ and $x_{s_{1}}=1-\frac{2 r}{\sigma^{2}}$. For $H>0$, we set $D_{H}=\{s \in \mathbf{R}: s \leq H\}$. By Theorem 3.1, $f\left(S_{T}\right) I_{\tau_{D_{H}}>T}$ can be hedged by the following:

$$
f\left(S_{T}\right) I_{\left\{S_{T}>H\right\}}-f\left(\frac{H^{2}}{S_{T}}\right)\left(\frac{S_{T}}{H}\right)^{1-\frac{2 r}{\sigma^{2}}} I_{\left\{S_{T}<H\right\}} .
$$

Now we notice that $p$ in (1.1) is $1-\frac{2 r}{\sigma^{2}}$.

\section{Acknowledgments}

The authors are grateful to Professors Jirô Akahori, for many valuable comments, careful reading the manuscript and suggesting several improvements. The authors wish to express their thanks to anonymous referee. 


\section{References}

Akahori, J., \& Y. Imamura (2012). On a Symmetrization of Diffusion Processes, arXiv:1206.5983v1 [q-fin.CP]

Akahori, J., \& K. TAKagi (2010). Static Hedging for Knock-in/out Options written on the Price Ratios: a Simple Case. in Proceedings of the 41st ISCIE International Symposium on Stochastic Systems Theory and its Applications, 201-205. Inst. Syst. Control Inform. Engrs. (ISCIE), Kyoto.

Biane, P., P. Bougerol, \& N. O'Connell (2005). Littelmann Paths and Brownian Paths. Duke Math. J., 130(1), 127-167

Bowie, J., \& P. CARr (1994). Static Simplicity. Risk, (7), 45-49

Carr, P., \& A. Chou (1997). Breaking Barriers. Risk, 10(9), 139-145

Carr, P., K. Ellis, \& V. Gupta (1998). Static Hedging of Exotic Options. Journal of Finance, 53(3), 1165-1196.

Carr, P., \& R. Lee (2009). Put-Call Symmetry: EXTENSIONS AND APPLICATIONS. Mathematical Finance, 19(4), 523-560

Grabiner, D. J. (1999). Brownian motion in a Weyl chamber, non-colliding particles, and random matrices. Ann. Inst. H. Poincaré Probab. Statist., $35(2), 177-204$

HALL, B. C. (2003). Lie groups, Lie algebras, and representations : an elementary introduction, Graduate Text in Mathematics, 222. SpringerVerlag, New York.

Humphreys, J. E. (1990). Reflection groups and Coxeter groups. Cambridge Studies in Advanced Mathematics, 29, Cambridge University Press, Cambridge.

Imamura, Y. (2011). A Remark on Static Hedging of Options Written on the Last Exit Time. Review of Derivatives Research, Volume 14, Number 3, 333-347.

Jones, L., \& N. O'Connell (2006). Weyl chambers, symmetric spaces and number variance saturation. ALEA Lat. Am. J. Probab. Math. Stat., $2,91-118$ 
Karlin, S., \& J. McGregor (1959). Coincidence probabilities. Pacific J. Math., 9, 1141-1164

Merton, R. C. (1973). Theory of Rational Option Pricing. Bell Journal od Economics and Management Science, 4, 141-183

Molchanov, I., \& M. Schmutz (2010). Multivariate Extension of PutCall Symmetry. SIAM Journal on Financial Mathematics, 1, 396-426

O'Connell, N. (2003). Random matrices, non-colliding processes and queues. in Séminaire de Probabilités, XXXVI, vol. 1801 of Lecture Notes in Math., 165-182. Springer, Berlin.

Poulsen, R. (2006). Barrier options and their static hedges: simple derivations and ex tensions. Quantitative Finance, 6(4), 327-335

Schmutz, M. (2011). Semi-static hedging for certain Margrabe-type options with barriers. Quantitative Finance, 11(2), 979-986 